\title{
Iterative resource allocation for ranking spreaders in complex networks
}

\author{
Zhuo-Ming Ren ${ }^{1,2}$, An Zeng ${ }^{2,3(a)}$, Duan-Bing Chen ${ }^{2,4}, \mathrm{HAO}_{\text {LiAO }}^{2}$ and JiAn-Guo Liu(b) \\ ${ }^{1}$ Research Center of Complex Systems Science, University of Shanghai for Science and Technology \\ Shanghai 200093, PRC \\ 2 Department of Physics, University of Fribourg - Chemin du Musée 3, CH-1700 Fribourg, Switzerland \\ 3 School of Systems Science, Beijing Normal University - Beijing 100875, PRC \\ 4 Web Sciences Center, University of Electronic Science and Technology of China - Chengdu 611731, PRC
}

\begin{abstract}
Ranking the spreading influence of nodes in networks is a very important issue with wide applications in many different fields. Various topology-based centrality measures have been proposed to identify influential spreaders. However, the spreading influence of a node is usually not only determined by its own centrality but also largely influenced by the centrality of neighbors. To incorporate the centrality information of neighbors in ranking spreaders, we design an iterative resource allocation (IRA) process in which the resource of nodes distributes to their neighbors according to neighbors' centrality. After iterations, the resource amount on each node will be stable and the final resources of nodes are used to rank their spreading influence. The iterative process can be applied to many traditional centrality measures including degree, K-shell, closeness, and betweenness. The validation of our method is based on the susceptible-infectedrecovered (SIR) spreading in four representative real datasets. The results show that the ranking accuracy of the traditional centrality measures is remarkably enhanced by IRA.
\end{abstract}

Introduction. - Spreading is one of the most successful application areas of the new science of networks [1-3]. It has application in many fields such as the virus spreading [4], reaction diffusion processes [5], pandemics [6], cascading failures [7] and so on. Many previous works were devoted to study it in the context of the nonequilibrium phase transitions $[8,9]$. In the past a few years, more and more attention has been paid to studying the spreading process in a microscopic way [10-12]. Among these topics, the identification of the most influential spreaders is an important one but still remains a challenge $[11,12]$ (for a short review, see [13]). With an effective algorithm to identify influential spreaders, we can, for instance, hinder spreading in the case of diseases or accelerate spreading in the case of information dissemination.

So far, various centrality measures have been applied to rank spreaders in complex networks. Related classical centrality measures include the degree as the number

\footnotetext{
(a) E-mail: an.zeng@unifr.ch

(b) E-mail: liujg004@ustc.edu.cn
}

of neighbors a node connects with, the closeness centrality [14] as the reciprocal of the sum of the geodesic distances to all other nodes, betweenness centrality [15] as the number of shortest paths through a certain node, eigenvector centrality [16] as the component of the eigenvector to the largest eigenvalue of the adjacency matrix, K-shell centrality [11] as the node location in a network. Lately, a lot of works have tried to design effective algorithms that outperform the classical centrality methods. For example, some algorithms focus on directly modifying the basic centrality measures including degree [17-19], closeness and betweenness [20-23]. Some works focus on improving the K-shell method by removing the degeneracy of the method [24-26]. Some others try to cut down the computational complexity of the eigenvector [27]. Moreover, the concept of path diversity is used to improve the ranking of spreaders [28]. Some methods are also designed in directed networks to identify the influential spreaders such as LeaderRank, which is shown to outperform the well-known PageRank method in both effectiveness and robustness [29]. 
The above classical or extended centralities could manifest different spreader topology in a network, which lead to different effectiveness and applicability for ranking the influence of the spreaders [30]. However, the spreading influence of a node is usually not only determined by its own centrality but also largely influenced by the centrality of its neighbors [31]. Therefore, it is necessary to develop an effective spreader ranking method which incorporates the centrality information of its own and neighbors. To this end, we design an iterative resource allocation (IRA) process for ranking spreaders in networks. Specifically, each node will start with one unit resource and diffuse the resource to all its neighbors according to the neighbors' centrality. After the resource on each node reaches the steady state, the amount of resource of nodes is used to rank their spreading influence. To evaluate the effectiveness of the proposed measure, we apply the susceptible-infectedrecovered (SIR) model to estimate the true spreading influence of nodes in four representative real networks. Kendall's tau coefficient is used to evaluate the correlation between the rank from different algorithms and the rank from the true spreading influence. The results show that the IRA method can significantly improve the ranking accuracy of the existing centrality measures.

Models. - We first describe four well-known centrality measures considered in this letter. The degree centrality is the simplest one, which is defined as the number of connections of a node. It is reasonable to assume the nodes with many connections to have stronger influence than those with few connections. In most cases, the degree is a powerful index for ranking the nodes' influence for its low computational cost. It works very well especially when the infection rate is small.

The closeness centrality [14] of node $i$ is defined as the reciprocal of the sum of the geodesic distances to all other nodes. Nodes with high closeness have short distances to others, and thus generally more influential in spreading. In the case of information diffusion, people usually consider individuals with high closeness value as being well positioned to obtain novel information early. Likewise, nodes with high closeness value in an epidemic network are positioned to infect others easily.

The betweenness centrality [15] of a node is defined as the fraction of the shortest paths in the network passing through it. Nodes with high betweenness is usually considered to have considerable influence. The closeness centrality and the betweenness centrality could effectively quantify the influence of nodes, but they are with high computational complexity due to calculating the shortest paths between all pairs of nodes in a network.

Kitsak et al. [11] presented a coarse-grained method by using K-shell decomposition to identify the influential nodes. The K-shell decomposition method $[32,33]$ could be implemented to identify the network shell in the following way. Firstly, it removes all nodes with degree one, and then keeps pruning the existed nodes until all nodes' degrees are larger than 1 . The removed nodes would form a node set whose $\mathrm{K}$-shell value is equal to one $(k s=1)$. In a similar way, it iteratively removes the next K-shell $(k s=2)$, and continues removing higher- $k$ shells until all nodes are removed. At the end of the decomposition, each node is associated with its own K-shell index, which indicates the topological location of a node. It should be noticed that the K-shell method assigns many nodes with the same K-shell value even though they perform differently in the spreading process.

We now introduce the iterative resource allocation (IRA) process. The basic idea is that each node has a unit resource as the initial configuration, and then diffuses the resource to its neighbors according to neighbors' centrality. After some iterations, the resource of each node will approach a steady state and the final amount of resource in each node will be used to rank the spreading influence of nodes. The IRA process can be detailedly described as follows.

An undirect network $G=(V, E)$ with $n=|V|$ nodes and $e=|E|$ links could be represented by an adjacent ma$\operatorname{trix} \Omega=\left\{\delta_{i j}\right\} \in R^{n, n}$, where $\delta_{i j}=1(i \neq j)$ if node $i$ and node $j$ are connected, and $\delta_{i j}=0$ otherwise. At each time step, resources in each node are allotted to its neighbors, and the allocation rule is determined by its neighbors' centrality. Denoting $J(i)$ as the set of node $i$ 's neighbors, the total resource node $i$ received will be

$$
\begin{aligned}
I_{i}(t+1)= & \sum_{j \in J(i)} R_{j \rightarrow i}(t+1)= \\
& \sum_{j \in J(i)}\left(\frac{\theta_{i}^{\alpha}}{\sum_{u \in J(j)} \theta_{u}^{\alpha}} \delta_{i j}\right) I_{j}(t),
\end{aligned}
$$

where $R_{j \rightarrow i}(t+1)$ is the amount of resource allotted from $j$ to $i$ at step $t+1$. $I_{j}(t)$ stands for the resources held by node $j$ at step $t$. $\theta_{i}$ is a certain centrality of the node $i$. The tunable parameter $\alpha$ is used to nonlinearly adjust the weight of the centrality. Equation (1) can be expressed in terms of a matrix as follows:

$$
I(t+1)=A I(t)=\left(\begin{array}{ccc}
a_{11} & \ldots & a_{1 n} \\
\vdots & \ddots & \vdots \\
a_{n 1} & \ldots & a_{n n}
\end{array}\right)\left(\begin{array}{c}
I_{1}(t) \\
\vdots \\
I_{n}(t)
\end{array}\right)
$$

where the elements of matrix $A$ are given by

$$
a_{i j}=\frac{\theta_{i}^{\alpha}}{\sum_{u \in J(j)} \theta_{u}^{\alpha}} \delta_{i j} .
$$

Since $a_{i j} \geq 0$ and $\sum_{i} a_{i j}=1$, the spectral radius $(\rho(A))$ of matrix $A$ (i.e., the largest absolute value of an eigenvalue) is no greater than 1 according to the Gershgörin disk theorem [34]. Furthermore, we can see that if $a_{i j}>0$, then $a_{j i}>0$. According to the PerronFrobenius theorem [34], $A^{t}$ converges to a constant matrix for infinite $t=t_{c}$, and the lower bound of $t_{c}$ is 


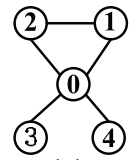

(a)

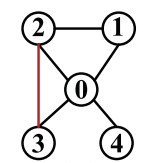

(b)

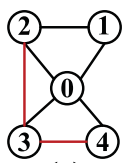

(c)

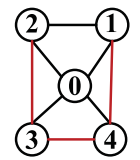

(d)
Fig. 1: (Color online) Example of networks with $N=5$. The node label is $0,1,2,3,4$, respectively. (a) $k s_{(0,1,2)}=2$, $k s_{(3,4)}=1$. (b) $k s_{(0,1,2,3)}=2, k s_{(4)}=1$. (c) $k s_{(0,1,2,3,4)}=2$. (d) $k s_{(0,1,2,3,4)}=3$.

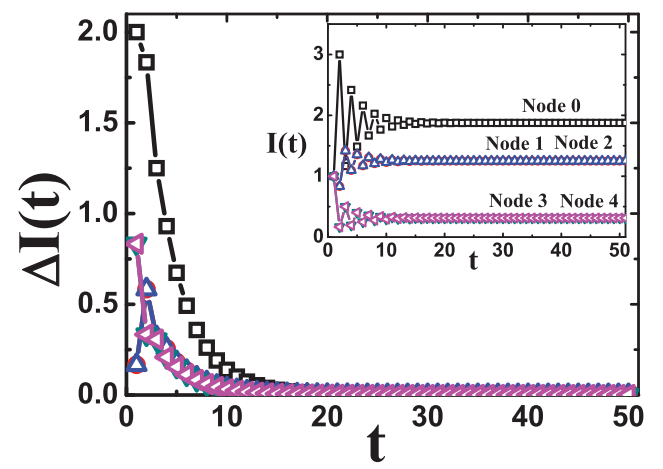

Fig. 2: (Color online) Illustration of the $\Delta I(t)$ value which evolves with iteration times $(t)$ on the small network in fig. 1(a). Inset: illustration of each node resource $I(t)$ value which evolves with iteration times $(t)$ on the small network.

determined $\left(t_{c} \leq 2 N-1\right)$. That is to say, we set small enough $\varepsilon(\varepsilon>0)$ as initial condition (we assume $\left.I(0)=(1,1, \cdots, 1)^{T}\right)$, then $I(t)$ will converge in the limit of infinite $t_{c}$ as $\left|I\left(t_{c}\right)-I\left(t_{c}-1\right)\right|<\varepsilon$. Consequently, it is clear that $I\left(t_{c}\right)$ is the dynamic equilibrium state of the resource allocation in our model. In practice, we can carry out the network allocation as follows:

i) Initialize resource of the network: $I(0)=$ $(1,1, \cdots, 1)^{T}$.

ii) Update the resource of each node in step $t$ based on $I(t)=A I(t-1)$ or $I(t)=A^{t} I(0)$.

iii) If $\Delta I(t)=|I(t)-I(t-1)|<\varepsilon\left(\varepsilon=10^{-6}\right.$ in this letter), terminate the iteration; otherwise, continue the iteration process according to ii).

As an example, a toy network with 5 nodes and 5 links is shown in fig. 1(a). The initial resource of each node equals $1\left(I(0)=[1,1,1,1,1]^{T}\right)$. We take the $\mathrm{K}$-shell as the node centrality $(\theta=k s$ as an example) and set $\alpha=1$. The resource allocation matrix is as follows:

$$
\mathbf{A}=\left(\begin{array}{ccccc}
0 & 1 / 2 & 1 / 2 & 1 & 1 \\
1 / 3 & 0 & 1 / 2 & 0 & 0 \\
1 / 3 & 1 / 2 & 0 & 0 & 0 \\
1 / 6 & 0 & 0 & 0 & 0 \\
1 / 6 & 0 & 0 & 0 & 0
\end{array}\right)
$$

To make the final resource allocation value convergent, we set large enough iteration steps $t_{c}=50$ and $I(50)=$ $A^{50} I(0)=[15 / 8,5 / 4,5 / 4,5 / 16,5 / 16]^{T}$. As shown in fig. 2 , the $\Delta I(t)$ value rapidly decreases as the iteration steps increase.
Table 1: The resource of each node in small networks shown in fig. 1 , where $\theta$ is the $\mathrm{K}$-shell value, $\alpha=1$, and the iteration times $t_{c}=50$.

\begin{tabular}{cccccc}
\hline \hline Network & 0 & 1 & 2 & 3 & 4 \\
\hline (a) & $\mathbf{1 . 8 8}$ & 1.25 & 1.25 & 0.31 & 0.31 \\
(b) & $\mathbf{1 . 5 9}$ & 0.91 & 1.36 & 0.91 & 0.23 \\
(c) & $\mathbf{1 . 4 3}$ & 0.71 & 1.07 & 1.07 & 0.71 \\
(d) & $\mathbf{1 . 2 5}$ & 0.94 & 0.94 & 0.94 & 0.94 \\
\hline \hline
\end{tabular}

To better understand the IRA method, we add some links to the toy network in fig. 1(a). We can observe that the degree, closeness and K-shell centralities of the node 0 are the same in fig. $1(\mathrm{a})-(\mathrm{d})$ (note that $k s$ of node 0 changes in $(d))$. However, the centralities of the neighboring nodes are changing as new links are added to the network. We set $\theta=k s$ in the IRA method as an example, the final resource of each node is presented in table 1 . One can clearly see that the resource of node 0 gradually decreases as new links are added to the network. This confirms that the IRA method can incorporate neighbors' centrality information when estimating the influence of the target node.

Data and metric. - In this letter, we test IRA method in four representative real networks: 1) Internet network at the autonomous system (AS) level. This network is collected by the archipelago active measurement infrastructure developed by the Cooperative Association for Internet Data Analysis [35]. Nodes represent autonomous systems (aggregations of Internet routers under the same administrative policy), and links represent the existence of border gateway protocol (BGP) peer connections between the corresponding autonomous systems. 2) Pretty-goodprivacy (PGP) network. This network is defined by the users of the PGP encryption algorithm for secure information exchange. Nodes represent users of the PGP algorithm [36]. A connection between two nodes indicates that each user has signed the encryption key of the other. 3) Enron email communication (Enron) network. The network covers all the email communication within a dataset of around half-million emails [37]. Nodes of the network are email addresses. If an address $i$ sends at least one email to address $j$, the network contains an undirected link between $i$ and $j$. 4) Condense matter physics collaboration (Cond-mat) network. The network covers scientific collaborations between authors [38]. If an author $i$ co-authors a paper with author $j$, the network contains an undirected link between $i$ and $j$. The relevant topological features of the different networks are summarized in table 2 .

Each method discussed above will generate a ranking list for nodes. In principle, the ranking from an effective ranking method should be as close as possible to the ranking based on the real spreading process. In this letter, we employ the SIR model [4] to simulate the spreading process on networks. In the SIR model, we set all nodes as initially susceptible except for one infectious node. At each 
Table 2: Topological features of the real network datasets considered: network node size $(N)$, network link size $(E)$, average degree $(\langle k\rangle)$, assortativity coefficient $(r)$ [39], the epidemic threshold $\left(\lambda_{c}\right)[40]$.

\begin{tabular}{ccccrc}
\hline \hline Data sets & $N$ & $E$ & $\langle k\rangle$ & \multicolumn{1}{c}{$r$} & $\lambda_{c}$ \\
\hline AS & 23752 & 58416 & 4.92 & -0.19 & 0.0034 \\
PGP & 10680 & 24316 & 4.55 & 0.25 & 0.0530 \\
Enron & 36692 & 183831 & 10.00 & -0.11 & 0.0071 \\
Cond-mat & 23133 & 93439 & 8.08 & 0.15 & 0.0453 \\
\hline \hline
\end{tabular}

step, the infected nodes will spread the virus to susceptible neighbors with a certain infectious rate $(\mu)$, and an infected node will recover after two spreading steps. The spreading influence of node $i$ is denoted as $S_{i}^{\mu}$, which is quantified in terms of the total prevalence of the epidemic process, i.e. the fraction of nodes being infected when the infection starts at node $i$. Based on this, we could obtain the true spreading influence of nodes and a corresponding ranking can be generated. We employ Kendall's tau coefficient [41] to measure the correlation between the topology-based ranking and the spreading-based ranking. Kendall's tau coefficient counts the difference between the number of concordant pairs and the number of discordant pairs of two rankings, according to

$$
\tau=\frac{\sum_{i \neq j} \operatorname{sgn}\left[\left(x_{i}-x_{j}\right)\left(y_{i}-y_{j}\right)\right]}{N(N-1)},
$$

where $\operatorname{sgn}(x)$ is the sign function, which returns 1 if $x>0$; -1 if $x<0$; and 0 if $x=0$. Here $\left(x_{i}-x_{j}\right)\left(y_{i}-y_{j}\right)>0$ means concordant, and a negative value means discordant. A higher Kendall's tau value indicates a more accurate ranking of the nodes' spreading ability.

\section{Numerical results. -}

Effectiveness of the IRA method. The IRA method requires the node centrality as the input. Here, we assign the node centrality $\theta$ as the degree $(k)$, K-shell $(k s)$, closeness $(C)$, and betweenness $(B)$, respectively. The final resources are received by the nodes based on these centralities which are respectively denoted as $I^{k}, I^{k s}, I^{C}$ and $I^{B}$. We start our analysis by considering the basic IRA method (i.e. $\alpha=1$ ). In fig. 3 , we report the improved ratio in $\tau$ when applying the IRA method to different centrality measures. The improved ratio is defined as $\eta=\frac{\tau^{\mathrm{IRA}}-\tau^{(0)}}{\tau^{(0)}}$, where $\tau^{(0)}$ is Kendall's tau value of a certain centrality method and $\tau^{\mathrm{IRA}}$ is the tau value after applying the IRA process to this centrality method. Clearly, $\eta>0$ indicates an advantage of the IRA method. As shown in fig. 3, one can find that the ranking accuracy has been considerably improved by the IRA method in different traditional centrality measurements. The largest $\eta$ for degree, K-shell, closeness, betweenness could reach 55\%, 35\%, 90\%, and $25 \%$, respectively.

Furthermore, we see the dependence of $\eta$ on the infectious rate in fig. 3 . We observe that $\eta$ has two distinct
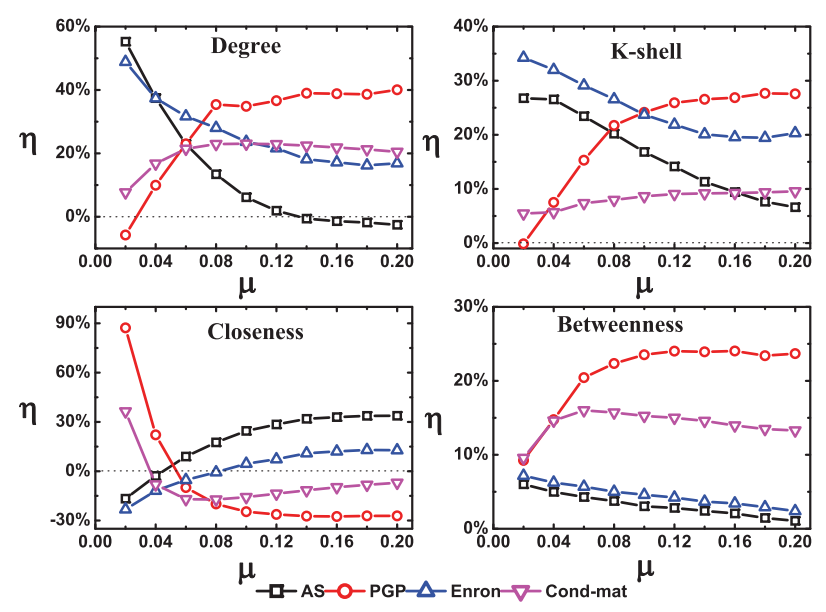

Fig. 3: (Color online) The improved ratio $(\eta)$ of different infectious rates $(\mu)$ with different centralities including degree, K-shell, closeness, and betweenness on AS, PGP, Enron, Condmat networks. The parameter $\alpha=1$ is fixed.

trends with the infectious rate in different networks, i.e., $\eta$ decreases with the infectious rate in the AS and Enron networks (with positive assortativity coefficient), while $\eta$ increases with the infectious rate in the PGP and Condmat networks (with negative assortativity coefficient) [39]. However, $\theta=C$ is a special setting for the IRA method. $\eta$ increases with the infectious rate in the assortative networks but decreases with the infectious rate in the disassortative networks. In PGP and Cond-mat networks, $\eta$ can be even lower than 0 , which we will discuss again below.

Breaking the ties in the ranking. Local ranking algorithms such as degree and K-shell have low ranking resolution, i.e., many nodes are ranked the same even though they actually have very different spreading influence [25]. Since many real networks have power-law degree distribution, the networks are usually dominated by small degree nodes. A large number of nodes cannot be distinguished if ranked by degree. This problem is even more serious in the K-shell method since it is a more coarse-grained method than degree.

We find that the IRA method can help these local ranking algorithms remove the degeneracy in the ranking. In fig. 4, we select all the nodes with the same $k$ and study their spreading influence $S^{\mu}$. The results show that $S^{\mu}$ of these nodes is significantly different from each other. However, the degree in this case is not an appropriate index to rank the spreading influence of these nodes since they are with the same degree. In this case, $\tau=0$ according to the definition of Kendall's tau. In fig. 4, we show that the IRA method can lead to $\tau>0$ when applied to ranking these nodes, which indicates that the ranking from IRA and the rank of the spreading ability are positively correlated. Therefore, the nodes with the same degree are well distinguished by the IRA method. We test different values of infectious rate $\mu$ and the results are consistent. A similar analysis is carried out for the K-shell method. 

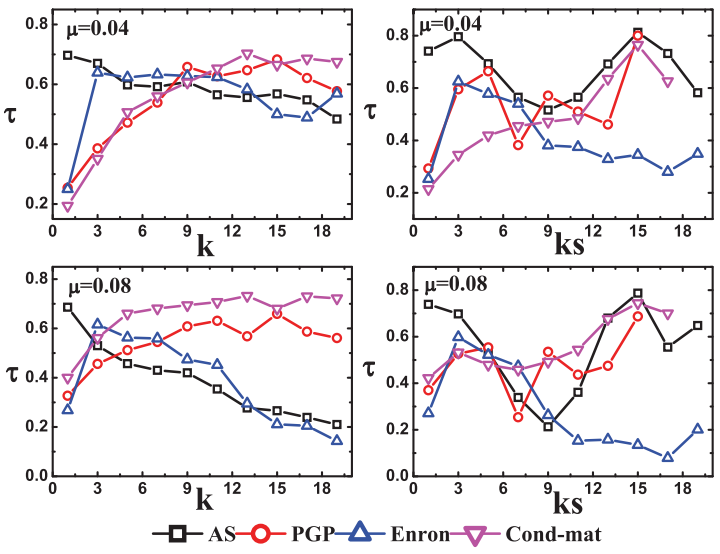

Fig. 4: (Color online) Kendall's $\tau$ between $S^{\mu}$ and $I^{k}\left(I^{k s}\right)$ of the nodes with the same $k(k s)$. Two representative infectious rates $\mu$ are selected for comparison.

Table 3: The improved ratio $\eta_{x}=\frac{\tau^{I^{k s}}-\tau^{(x)}}{\tau^{(x)}}$, where $\tau^{(x)}$ is Kendall's tau value of a certain centrality method and $\tau^{I^{k s}}$ is the tau value of the IRA method with $\theta=k s . \alpha^{*}$ is the optimal parameter for the $I^{k s}$ ranking according to fig. 5 , and the infectious rate $(\mu)$ is 0.04 .

\begin{tabular}{cccccc}
\hline \hline Networks & $\alpha^{*}$ & $\eta_{k}(\%)$ & $\eta_{k s}(\%)$ & $\eta_{C}(\%)$ & $\eta_{B}(\%)$ \\
\hline AS & 4 & 37.4 & 33.1 & 9.6 & 88.9 \\
PGP & 1.5 & 4.9 & 7.5 & 21.3 & 52.7 \\
Enron & 3 & 49.6 & 43.9 & 9.8 & 69.6 \\
Cond-mat & 5 & 18.7 & 14.3 & 1.6 & 78.5 \\
\hline \hline
\end{tabular}

In fig. 4, we also can see that a high $\tau$ can be achieved as well in different $k s$ values.

We remark that the monotonic ranking in the IRA method occurs because it incorporates the centrality of neighbors. If a node is connecting to neighbors with high centrality, the spreading initialized from it will finally have wider coverage than the nodes with the same $k$ (or $k s$ ) but connecting to lower centrality neighbors.

Improvement from the tunable parameter. In this subsection, we will discuss the effect of the tunable parameter $\alpha$ in the IRA method. We fix the infectious rate as 0.04 and show the results in fig. 5. One can observe that there exists an optimal $\alpha$ when IRA is applied to each centrality. Interestingly, the optimal $\alpha$ is around 0 in the betweenness case and its $\tau$ is relatively low, which indicates that the betweenness is not a suitable centrality to be used in the IRA method. Generally speaking, the closeness is the best centrality for IRA since it can achieve the highest $\tau$ value. However, since the closeness is with high computation complexity, one can use the degree or K-shell measure as an alternative centrality in the IRA method. They can lead to a similar $\tau$ as the closeness when used in the IRA method, but they are much faster than closeness to calculate. In table 3 , we report the improved ratio $\eta$ of the $I^{k s}$ method to the traditional centrality methods as an example. One can easily see that the $I^{k s}$ considerably outperforms these traditional centrality methods. We remark
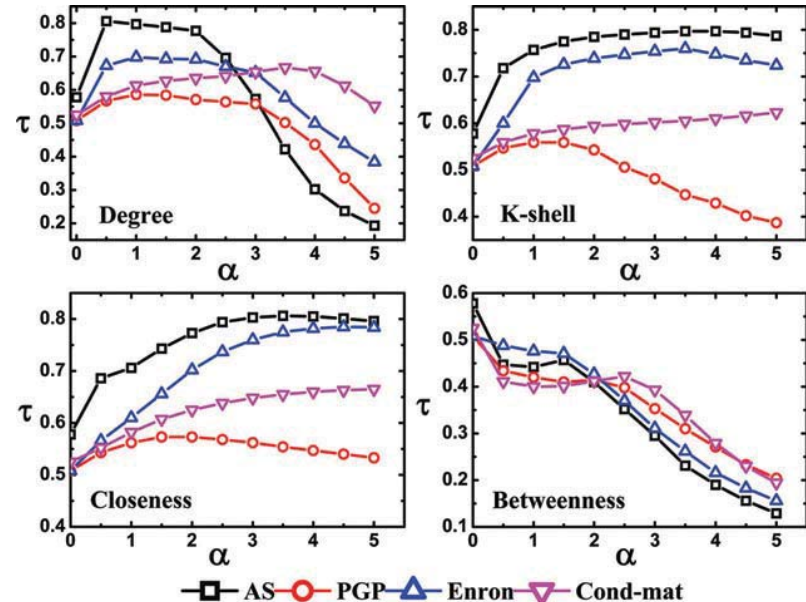

Fig. 5: (Color online) The performance $(\tau)$ of different parameters $\alpha$ with different centralities including degree, K-shell, closeness, and betweenness on AS, PGP, Enron, Cond-mat networks. The infectious rate $(\mu)$ is 0.04 .
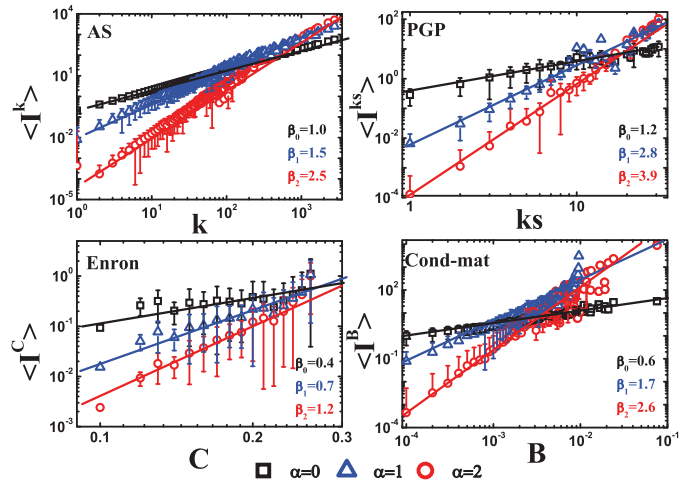

Fig. 6: (Color online) The correlation between influence $\left\langle I^{\theta}\right\rangle$ and node centralities $\theta$ on AS, PGP, Enron, Cond-mat networks with different $\alpha$. The $\beta$ is the estimated exponent and the error bars denote the standard deviation of $I^{\theta}$ of the nodes with the same $\theta$.

that the optimal $\alpha$ still exists when the infectious rate is larger. Moreover, for negative $\eta$ in the closeness case in fig. 3, optimal $\alpha$ can finally lead to positive $\eta$.

The distribution of resource in the IRA method. Finally, we analyze the distribution of resource in the IRA method under different values of parameter $\alpha$. Taking the degree as an example, we average $I^{k}$ of the nodes with the same $k$, and study the correlation of $\left\langle I^{k}\right\rangle$ and $k$. For other centrality measures, we also do the same procedure and report the results in fig. 6 . It shows that the nodes with high $k$ could have high resource $\left\langle I^{k}\right\rangle$. Interestingly, there seems to be a positive correlation as $\left\langle I^{\theta}\right\rangle \sim \theta^{\beta}$ in all centrality cases. One can see that $\beta$ nonlinearly increases with $\alpha$, which indicates that the final resource distribution is nontrivially controlled by $\alpha$. Moreover, the error bars as the standard deviation of $I^{\theta}$ are shown in fig. 6 . The big error bar indicates that $I^{\theta}$ of the nodes with the same $\theta$ are well separated, thus leading to the breaking of the degeneracy. 
Conclusions and discussions. - In summary, we propose an iterative resource allocation method to rank the spreader spreading influence. It can be applied to many classical centrality measures such as degree, K-shell, closeness, betweenness and can improve the performance of them, i.e. by enhancing their accuracy in ranking spreaders. The simulation results show that the performance of the IRA can be further improved when an optimal parameter is assigned. Our results might also find practical applications in optimized immunization strategies, which can be designed to monitor the actual spreaders. In a broader context, our work could be relevant to other fields of spreading processes, such as information, behavior, rumor spreading or other dynamical processes, which may provide insights in the analysis of these collective behavior, from social influence to biomedical responses.

This work may lead to many extensions. In the IRA method, the final resource of a node is not only determined by its centrality but also by its neighbors' centrality. It would be very interesting to test the modified centrality in other dynamic process. For example, one can investigate whether the malicious attack according to the IRA-based degree will cause more harmful results to the giant component of networks $[42,43]$. In addition, the interdependent networks have attracted much attention recently [44]; how to design an iterative resource allocation in these systems can also be an interesting and important open problem.

We thank Prof. YI-CHeng ZHANG for fruitful discussion. This work is partially supported by the National Natural Science Foundation of China (Nos. 61374177, 71371125, 71171136), Shanghai Leading Academic Discipline Project (Systems Science) (No. XTKX2012), and the Special Project of Sichuan Youth Science and Technology Innovation Research Team (2013TD0006).

\section{REFERENCES}

[1] Ginsberg J., Mohebbi M. H., Patel R. S., Brammer L., Smolinski M. S. and Brilliant L., Nature, 457 (2008) 1012.

[2] Wang P., González M. C., Hidalgo C. A. and BarabÁsi A. L., Science, 324 (2009) 1071.

[3] Centola D., Science, 329 (2010) 1194.

[4] Keeling M. J. and Rohani P., Modeling Infectious Diseases in Humans and Animals (Princeton University Press) 2008.

[5] Aral S. and Walker D., Science, 337 (2012) 337.

[6] Pastor-Satorras R., Vázquez A. and Vespignani A., Phys. Rev. Lett., 87 (2001) 258701.

[7] Buldyrev S. V., Parshani R., Paul G., Stanley H. E. and Havlin S., Nature, 464 (2010) 1025.

[8] Newman M. E. J., Phys. Rev. Lett., 95 (2005) 108701.

[9] Marro J. and Dickman R., Nonequilibrium Phase Transitions in Lattice Models. (Cambridge University Press) 1999.

[10] Zeng A., J. Stat. Mech. (2013) P11010.
[11] Kitsak M., Gallos L. K., Havlin S., Lilueros F., Muchnik L., Stanley H. E. and Makse H. A., Nat. Phys., 6 (2010) 888.

[12] Ghoshal G. and Barabási A. L., Nat. Commun., 2 (2011) 394.

[13] Pei S. and Makse H. A., J. Stat. Mech. (2013) P12002.

[14] Sabidussi G., Psychometrika, 31 (1966) 581.

[15] Goh K. I., Oн E., Kahng B. and Kim D., Phys. Rev. E, 67 (2003) 017101.

[16] Borgatti S. P., Soc. Netw., 27 (2005) 55.

[17] Ugander J., Backstrom L., Marlow C. and KleinBerg J., Proc. Natl. Acad. Sci. U.S.A., 109 (2012) 5962.

[18] Chen D. B., LüL. Y., Shang M. S., Zhang Y. C. and Zhou T., Physica A, 391 (2012) 1777.

[19] Chen D. B., Gao H., LüL. Y. and Zhou T., PLoS ONE, 8 (2013) e77455.

[20] Dangalchev C., Physica A, 365 (2006) 556.

[21] Zhang J., Xu X. K., Li P., Zhang K. and Small M., Chaos, 21 (2011) 016107.

[22] Travencolo B. A. N. and Da F. Costa L., Phys. Lett. A, 373 (2008) 89.

[23] Comin C. H. and DA F. Costa L., Phy. Rev. E, 84 (2011) 056105.

[24] Garas A., Schweitzer F. and Havlin S., New J. Phys., 14 (2012) 083030.

[25] Zeng A. and Zhang C. J., Phys. Lett. A, 377 (2013) 1031.

[26] Liu J. G., Ren Z. M. and Guo Q., Physica A, 392 (2013) 4154.

[27] Poulin R., Boily M. C. and Masse B. R., Soc. Netw., 22 (2000) 187.

[28] Chen D. B., Xiao R., Zeng A. and Zhang Y. C., EPL, 104 (2014) 68006.

[29] Lü L. Y., Zhang Y. C., Yeung C. H. and Zhou T., PloS ONE, 6 (2011) e21202.

[30] DA Silva R. A. P., Viana M. P. and DA Fontoura Costa L., J. Stat. Mech. (2012) P07005.

[31] Ou Q., Jin Y. D., Zhou T., Wang B. H. and Yin B. Q., Phys. Rev. E, 75 (2007) 021102.

[32] Dorogovtsev S. N., Goltsev A. V. and Mendes J. F. F., Phys. Rev. Lett., 96 (2006) 040601.

[33] Carmi S., Havlin S., Kirkpatrick S., Havitt S. Y. and Shir E., Proc. Natl. Acad. Sci. U.S.A., 104 (2007) 11150 .

[34] Horn R. A. and Johnson C. R., Matrix Analysis (Cambridge University Press, Cambridge) 1985.

[35] Claffy K., Hyun Y., Keys K., Fomenkov M. and Krioukov D., in Proceedings of CATCH 2009, Washington, DC, March 2009 (IEEE) 2009, p. 205.

[36] Boguñám., Pastor-Satorras R., Díaz-Guilera A. and Arenas A., Phys. Rev. E, 70 (2004) 056122.

[37] Leskovec J., Lang K. J., Dasgupta A. and Mahoney M. W., Internet Math., 6 (2009) 29.

[38] Leskovec J., Kleinberg J. and Faloutsos C., ACM Trans. Knowl. Discov. (TKDD), 1 (2007) 2.

[39] Newman M. E. J., Phys. Rev. Lett., 89 (2002) 208701.

[40] Castellano C. and Pastor-Satorras R., Phys. Rev. Lett., 105 (2010) 218701.

[41] Kendall M., Biometrika, 30 (1938) 81.

[42] Zeng A. and Cimini G., Phys. Rev. E, 85 (2012) 036101.

[43] Zeng A. and Liu W., Phys. Rev. E, 85 (2012) 066130.

[44] Buldyrev S. V., Parshani R., Paul G., Stanley H. E. and Havlin S., Nature, 464 (2010) 1025. 